\title{
Gradient Wetting Transition from the Wenzel to Robust Cassie-Baxter States along Nanopillared Cicada Wing and Underlying Mechanism
}

\author{
Heng Xie, Hanxiong Huang* \\ Lab for Micro Molding and Polymer Rheology, Guangdong Provincial Key Laboratory of Technique and Equipment for Macromolecular \\ Advanced Manufacturing and Key Laboratory of Polymer Processing Engineering (Ministry of Education), \\ South China University of Technology, Guangzhou 510640, China
}

\begin{abstract}
Gradient wettability is important for some living organisms. Herein, the dynamic responses of water droplets impacting on the surfaces of four regions along the wing vein of cicada Cryptotympana atrata fabricius are investigated. It is revealed that a gradient wetting behavior from hydrophilicity (the Wenzel state) to hydrophobicity and further to superhydrophobicity (the Cassie-Baxter state) appears from the foot to apex of the wing. Water droplets impacting on the hydrophilic region of the wing cannot rebound, whereas those impacting on the hydrophobic region can retract and completely rebound. The hydrophobic region exhibits robust water-repelling performance during the dynamic droplet impact. Moreover, a droplet sitting on the hydrophobic region can recover its spherical shape after squeezed to a water film as thin as $0.45 \mathrm{~mm}$, and lossless droplet transportation can be achieved at the region. Based on the geometric parameters of the nanopillars at the hydrophilic and hydrophobic regions on the cicada wing, two wetting models are developed for elucidating the mechanism for the gradient wetting behavior. This work directs the design and fabrication of surfaces with gradient wetting behavior by mimicking the nanopillars on cicada wing surface.
\end{abstract}

Keywords: cicada wing, nanopillared surface, biomimetic, gradient wetting transition, dynamic wetting behavior Copyright $(\odot)$ The author(s) 2020.

\section{Introduction}

Natural creatures have experimented with various solutions to survive and the successful ones have evolved into unusual structures on their surfaces to help them survive in tough conditions ${ }^{[1-3]}$. The researches on biological surface structures and their functions have become one of the most attractive areas in bionics. For example, compound eyes of mosquito possess ideal superhydrophobicity for maintaining clear vision in a humid habitat ${ }^{[4]}$. Spiders capture water from humid air by using its silk with periodic wetting behavior ${ }^{[5]}$. Lizards render a gradient hydrophilic state along its body for collecting water from moisture ${ }^{[6]}$. The gradient surface roughness and Laplace pressure along the spine of cactus endow it with an efficient fog collection system ${ }^{[7]}$. The dynamics of water droplets on snail shell is strongly affected by the roughness and chemical heterogeneity on ${ }^{i t^{[8]}}$. Some animals and plants use micro/nano sized hydrophobic and hydrophilic patterns on their backs to capture water from humid air or fog ${ }^{[9,10]}$.
Gradient surfaces are important for some living organisms ${ }^{[11]}$. Inspired by the aforementioned natural systems containing surface gradient or diversity that enables water condensation or transport, micro/nanostructures with gradients in surface energy or gradients in Laplace pressure have been developed ${ }^{[11-13]}$. Consequentially, liquid behavior manipulation by structure-inducing surface gradient has become an area of interest because of its potential applications in oil/water separation, water collection, microfluidics, self-cleaning, and liquid transportation ${ }^{[14-16]}$. Although great progress has been made toward developing biomimetic gradient surfaces, there are still challenges in fabrication methods and liquid manipulation efficiency which impede their applications. The foremost challenge lies in constructing simple and efficient micro/nanostructures with gradient wetting behavior by low-cost and industrialized approaches. Fortunately, the ingenious surfaces in nature offer an ideal source of innovation for novel nano/microstructures with gradient wetting behavior.

\footnotetext{
*Corresponding author: Hanxiong Huang

E-mail: mmhuang@scut.edu.cn
} 
Cicada wings possess remarkable properties such as optical trapping ${ }^{[17,18]}$, hydrophobicity ${ }^{[19]}$, bacteria killing $^{[20,21]}$, biofouling prevention ${ }^{[22]}$ and condensate microdrop self-propelling ${ }^{[23-25]}$ due to packed nanopillars with nanoscale interspaces on their surfaces. As a flying insect, cicada has a camouflage ability associated with the antireflective property of its wing, which avoids it to be captured ${ }^{[26]}$. Moreover, cicada inevitably contends with rain, dew, fog, and so on. The surroundings cause problems for cicadas, both during flight and at rest. Fortunately, cicada wing can maintain a light weight and easily remove the accumulated water droplet from its surface by a slight shaking ${ }^{[27]}$. So, cicada wing can be used as a typical biological prototype for fabricating nanostructured surfaces with antireflective property $^{[28-30]}$, substrates for surface-enhanced Raman scattering ${ }^{[31,32]}$, and platforms for random lasing ${ }^{[33]}$. Despite researches have been performed on the nanostructures and static wetting behaviors of cicada family ${ }^{[19,23-25,30]}$, the reports on the dynamic wetting behavior on cicada wing and the dimensions of the nanostructures on the whole surface of a cicada wing are sparse to date.

A gradient static wetting behavior along the cicada wing vein of Cryptotympana atrata fabricius has been revealed in our previous work ${ }^{[27]}$. Further, responses of the water droplets impacting on the surface of the cicada wing of Cryptotympana atrata fabricius are investigated to evaluate the dynamic wetting behaviors along its wing vein in this work. The mechanism for the gradient wetting behavior on the cicada wing surface, which is vital for the applications of cicada wing inspired surfaces, is elucidated for the first time.

\section{Experimental section}

\subsection{Natural wing treatment}

The cicada (Cryptotympana atrata fabricius) wings were pretreated cautiously to ensure the accuracy and efficiency of impact and wetting state tests. Specifically, the wings were first washed by ultrasonic oscillation for 20 min with deionized water to remove contaminants absorbed physically on their surfaces. They were then ultrasonically cleaned in acetone for $20 \mathrm{~min}$ to remove the stains, proteins, and fattiness that were stuck to the pillars. Finally, they were ultrasonically cleaned in the deionized water again for $10 \mathrm{~min}$ to remove residual acetone, followed by drying in a vacuum oven at $40{ }^{\circ} \mathrm{C}$.

\subsection{Characterization and dynamic impact test}

Droplet impact tests (as shown in Fig. 1) were carried out at a relative humidity of $60 \% \pm 5 \%$ and room temperature of $25{ }^{\circ} \mathrm{C} \pm 1{ }^{\circ} \mathrm{C}$ to evaluate the dynamic wetting behavior on the cicada wings. Water droplets with an initial diameter $(D)$ of approximately $3 \mathrm{~mm}$ were generated from a precision syringe to drip on the wings. The velocity $(V)$ of the droplet dropping at a height of $l$ can be calculated according to the following equation:

$$
V=\sqrt{2 \mathrm{~g} l}
$$

where $g$ is the acceleration of gravity $\left(9.8 \mathrm{~m} \cdot \mathrm{s}^{-2}\right)$. The impact processes of the droplets on the wing surfaces were recorded using a high-speed camera (TS5, Fastec Imaging Corporation, USA) at $250 \mathrm{fps}$.

The cicada wings were coated with gold by ion sputtering, and were then observed by using a Scanning Electronic Microscopy (SEM; Nova NanoSEM 430, FEI Co., the Netherlands) to characterize the nanostructures on them. The Contact Angles (CAs) and Roll-off Angles (RAs) of $4 \mu \mathrm{L}$ droplets on the wing surfaces were measured using an automatic contact-angle testing apparatus (OCA 40, Data Physics Corporation, Germany). Both the CA and RA measurements were conducted at three different locations on the wings to obtain their average values.

\section{Results and discussion}

\subsection{Droplet impact behaviors on cicada wings}

The impact tests for a droplet ( $\sim 3 \mathrm{~mm}$ in diameter)

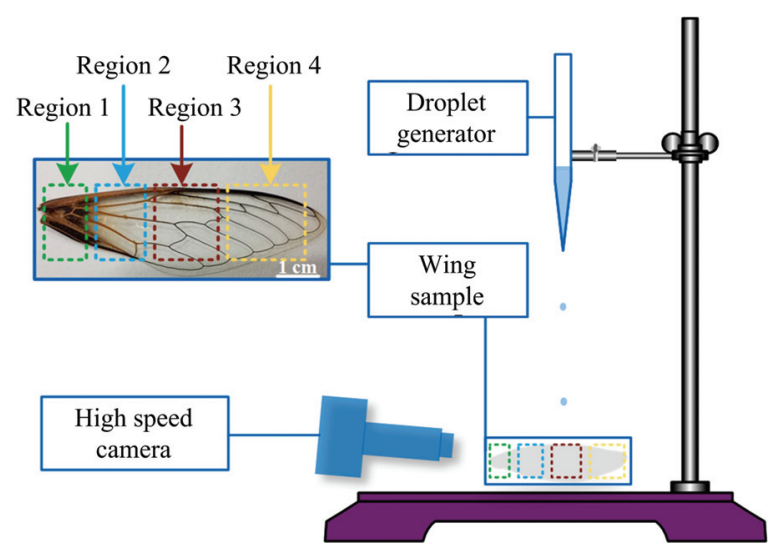

Fig. 1 Schematics of droplet impact test. 

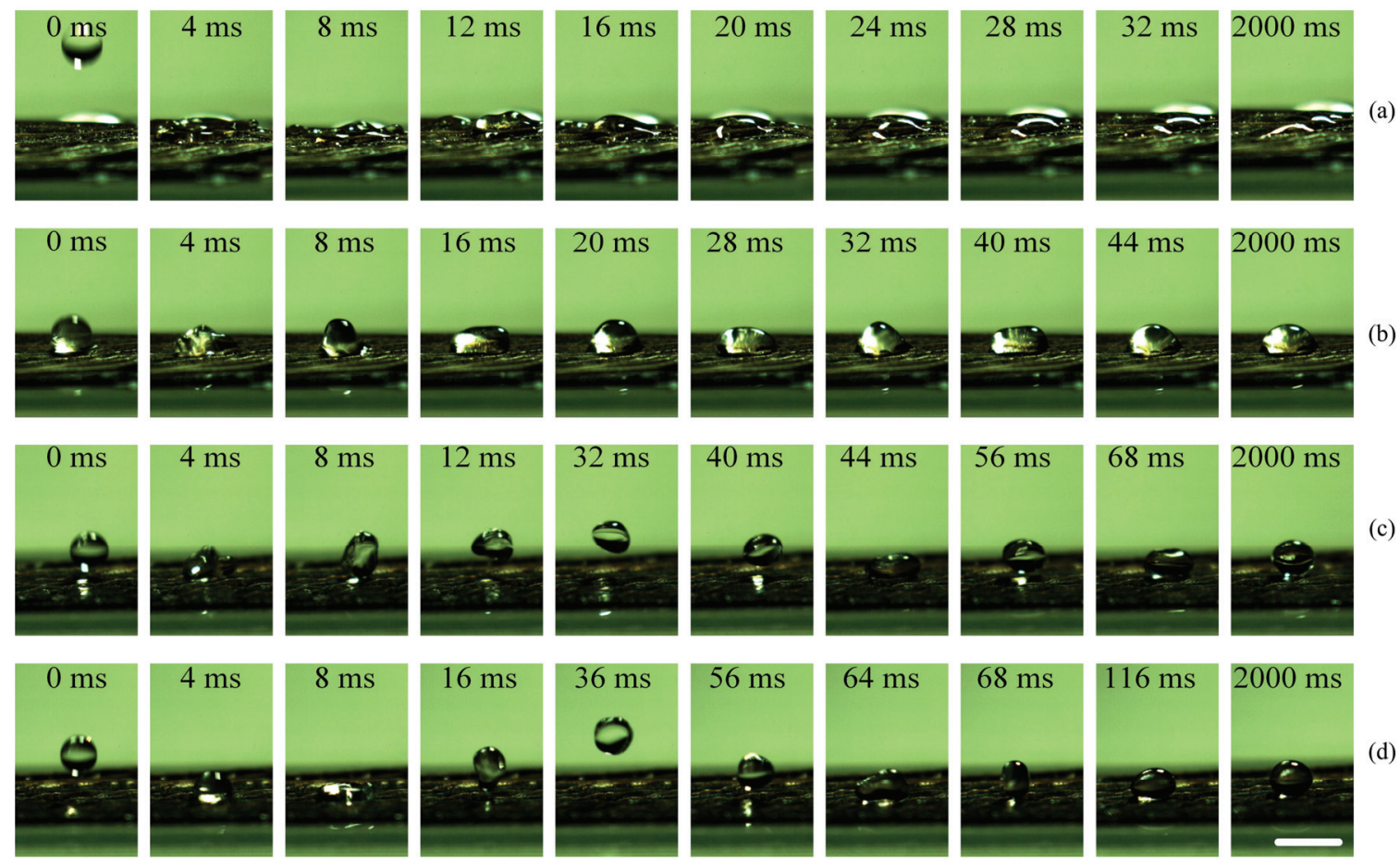

Fig. 2 Typical snapshots of droplet impacting at regions (a) 1, (b) 2, (c) 3, and (d) 4 along cicada wing vein (Impact velocity: $0.9 \mathrm{~m} \cdot \mathrm{s}^{-1}$; scale bar: $5 \mathrm{~mm}$ ).

dropping at a $40 \mathrm{~mm}$ height (corresponding to an impact velocity of $0.9 \mathrm{~m} \cdot \mathrm{s}^{-1}$ ) were performed at regions $1-4$ along the cicada wing veins (as shown in Fig. 1). The droplet impact processes at the four regions were recorded and are displayed in Supplementary Videos 1-4, and the typical snapshots taken from Videos $1-4$ are shown in Fig. 2.

As can be seen, after impacting at regions 1 and 2, the spherical droplet deforms first and spreads rapidly owing to a rapid increase in pressure at the point of impact $^{[34]}$. The droplet penetrates into the bottom of the inter-nanopillar areas and reaches the maximum spreading range more quickly at region 1 than that at region 2. The droplet impacting at region 2 undergoes viscous dissipation ${ }^{[35]}$ and oscillates several times until its energy is exhausted at $\sim 2000 \mathrm{~ms}$. Finally, the droplet stands at regions 1 and 2, both of which exhibit hydrophilicity. The difference lies in that the droplet has an obviously larger spread area at region 1 (Fig. 2a) and the droplet preserves the spherical shape finally at region 2 (Fig. 2b). That is, region 1 is more hydrophilic than region 2. After impacting at regions 3 and 4 , the droplet spreads to a small extent. Then, it retracts and com- pletely rebounds from the surfaces of regions 3 and 4 at about $8 \mathrm{~ms}$ and $16 \mathrm{~ms}$, respectively. The spreading, retracting, and rebounding of the droplet occur for two times. Finally, the droplet suspends on the surfaces with spherical shape, which means that regions 3 and 4 exhibit obvious hydrophobicity. The rebounding height of the droplet at region 4 is larger than that at region 3 in the first rebounding. The dynamic impact behaviors of the droplet at regions 1 to 4 demonstrate that the cicada wing surface exhibits different wetting behaviors along its vein.

Further, the hydrophobic region is impacted with a droplet at a higher impact velocity $\left(1.4 \mathrm{~m} \cdot \mathrm{s}^{-1}\right)$. The droplet impact process at region 3 is displayed in Supplementary Video 5, and the typical snapshots are shown in Fig. 3. As can be seen, after impacting the surface, the droplet quickly spreads and develops into pancake-like lamella with a wavy perimeter at $\sim 4 \mathrm{~ms}$ due to a rapid increase in pressure at the point of impact. Some small satellite droplets are separated from the edge of the droplet lamella in the spreading stage (at $\sim 8 \mathrm{~ms}$ ). Then, the main droplet retracts quickly, and the separated satellite droplets splash on the wing surface $(8 \mathrm{~ms}-20 \mathrm{~ms}$, 

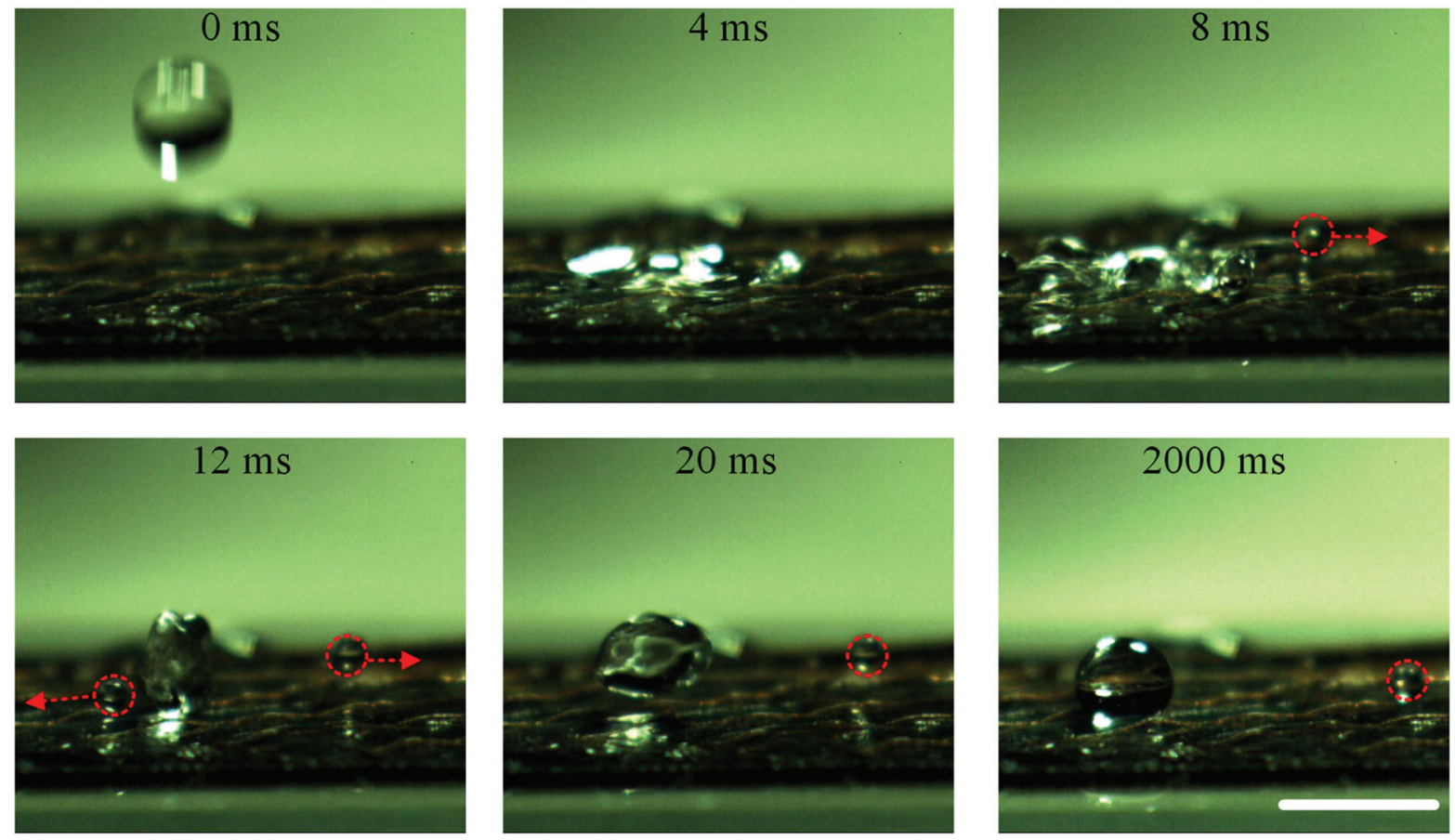

Fig. 3 Typical snapshots of droplet impacting on surfaces at hydrophobic region of cicada wing (Impact velocity: $1.4 \mathrm{~m} \cdot \mathrm{s}^{-1} ; \mathrm{scale}$ bar: $5 \mathrm{~mm})$.

marked by red dotted circles). Finally, the main droplet and separated satellite droplets suspend on the surface with spherical shape (at $\sim 2000 \mathrm{~ms}$ ). The aforementioned phenomenon results from the accumulation and viscous dissipation of the kinetic energy of the droplet. Thus, the Weber number $(\mathrm{We})^{[36]}$, the ratio of kinetic energy to surface energy, is calculated to characterize the impact behaviors:

$$
W e=\frac{\rho V^{2} D}{\gamma},
$$

where $\rho, D$, and $\gamma$ are the density $\left(1000 \mathrm{~kg} \cdot \mathrm{m}^{-3}\right)$, initial diameter, and surface tension $\left(0.072 \mathrm{~N} \cdot \mathrm{m}^{-1}\right.$ at $25{ }^{\circ} \mathrm{C} \pm$ $1{ }^{\circ} \mathrm{C}$ ) of the water droplet, respectively. For the droplets with the impact velocities of $0.9 \mathrm{~m} \cdot \mathrm{s}^{-1}$ and $1.4 \mathrm{~m} \cdot \mathrm{s}^{-1}$, the calculated $W e$ values are 33.75 and 81.67 , respectively. Some satellite droplets are separated from the impact droplet with the higher impact velocity to reduce the kinetic energy of the main droplet, and thus its rebounding height is decreased. Finally, the main and satellite droplets on the wing surface still exhibit a hydrophobic state. This means that the droplet with higher impact kinetic energy still does not wet the hydrophobic region (regions 3 and 4) on the cicada wing. The dy- namic pressure $\left(P_{\mathrm{D}}=\rho V^{2} / 2\right)$ of the droplet with the impact velocity of $1.4 \mathrm{~m} \cdot \mathrm{s}^{-1}$ is calculated to be $980 \mathrm{~Pa}$, indicating that the hydrophobic regions on the wing exhibit robust water-repelling performance during the dynamic droplet impact.

\subsection{Robust hydrophobic wetting state}

Squeezing a water droplet between two surfaces can be employed to investigate the stability of hydrophobic wetting state on surface against external pressure $^{[37,38]}$. The whole process of squeezing a $4 \mu \mathrm{L}$ droplet dropped at region 3 on the cicada wing is displayed in Supplementary Video S6, in which a polypropylene replica with hierarchical microstructure and low adhesion on its surface $\left(\mathrm{CA}=162^{\circ}, \mathrm{RA}<30^{\circ}\right)$ prepared in our previous work $^{[39]}$ is used to squeeze the droplet. The representative images taken from Video $\mathrm{S} 6$ are shown in Fig. 4a. The replica slowly approaches the top of the droplet dropped at region 3 on the wing, squeezing it to a certain extent (Fig. 4a-1-3). Then the replica is lifted slowly (Fig. 4a-4). The process of squeezing the droplet is repeated (Fig. 4a-5 and 6), in which the droplet is squeezed to a greater extent for $3 \mathrm{~s}$. Finally, the replica is removed upward slowly to relax the droplet (Fig. 4a-7). 


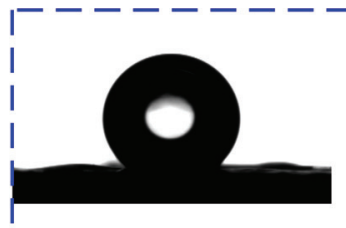

(1)

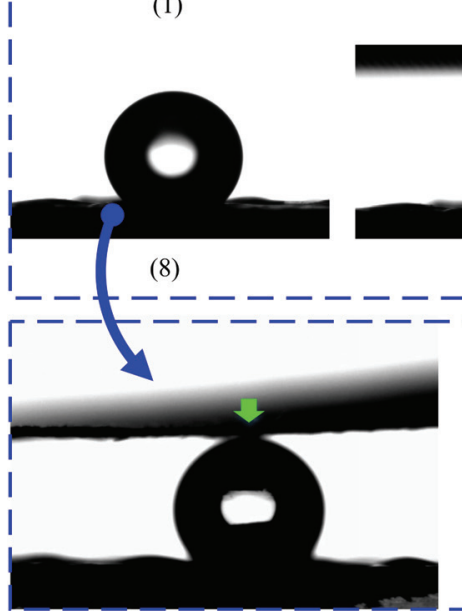

Fig. 4 (a) Representative images of $4 \mu \mathrm{L}$ droplet (1) before, $(2-6)$ during, and $(7,8)$ after being squeezed at region 3 on cicada wing (arrows represent moving direction of polypropylene replica); (b) typical images taken from transference process of squeezed and recovered droplet in (a) (arrow represents moving direction of filter paper).

Although the droplet is squeezed twice, it can recover its spherical shape (Fig. 4a-8). The aforementioned squeezing tests are also performed at five different locations at regions 3 and 4 on the wing surface, and the results demonstrate that all the squeezed droplets can recover their spherical shape. This means that the hydrophobic region on the wing can stabilize the non-wetting state of the droplet against the external pressure. Pressure difference $(\Delta P)$ generated during squeezing the droplet on the surface can be calculated using the following Laplace equation ${ }^{[40]}$ :

$$
\Delta P=\frac{\gamma\left|\left(\cos \theta_{\mathrm{t}}+\cos \theta_{\mathrm{w}}\right)\right|}{h}, \text { for } h<<D
$$

where $\theta_{\mathrm{t}}$ and $\theta_{\mathrm{w}}$ are the CAs of the water droplet on the replica and wing surfaces, respectively; $h$ is the gap between the surfaces of the replica and wing. The $\Delta P$ is calculated to be about $285.4 \mathrm{~Pa}$ at $h_{2}$ of $0.45 \mathrm{~mm}$ (Fig. 4a-6), which implies that the hydrophobic region on the wing is robust enough to stabilize the hydrophobicity under external pressure.

Further, the aforementioned squeezed and recovered $4 \mu \mathrm{L}$ droplet at region 3 on the wing is contacted by a filter paper downward. As can be seen in Supple- mentary Video S7 and Fig. 4b, the droplet completely adheres to the filter paper and then spreads out instantaneously once they touch each other, indicating the achievement of lossless droplet transportation.

\subsection{Mechanism analyses on gradient wetting be- havior along wing vein}

The phenomena observed in section 3.1 indicate that the water droplets exhibit different wetting states and a gradient wetting behavior from hydrophilicity to hydrophobicity and further to superhydrophobicity appears along the wing vein. It was demonstrated that the contribution of the nanopillars and their distribution to the wettability on cicada wings is more pronounced than that of the surface chemistry ${ }^{[30]}$. Therefore, the wetting behaviors at regions $1-4$ and the corresponding topographies are dissected to reveal the mechanism of the different wetting states along the wing vein in the follows.

The SEM images for the nanopillars at regions $1-4$ on the cicada wing surface are shown in Fig. 5, in which the wetting states for the water droplets with volumes of about $8 \mu \mathrm{L}-10 \mu \mathrm{L}$ and the CAs for $4 \mu \mathrm{L}$ water droplet at 


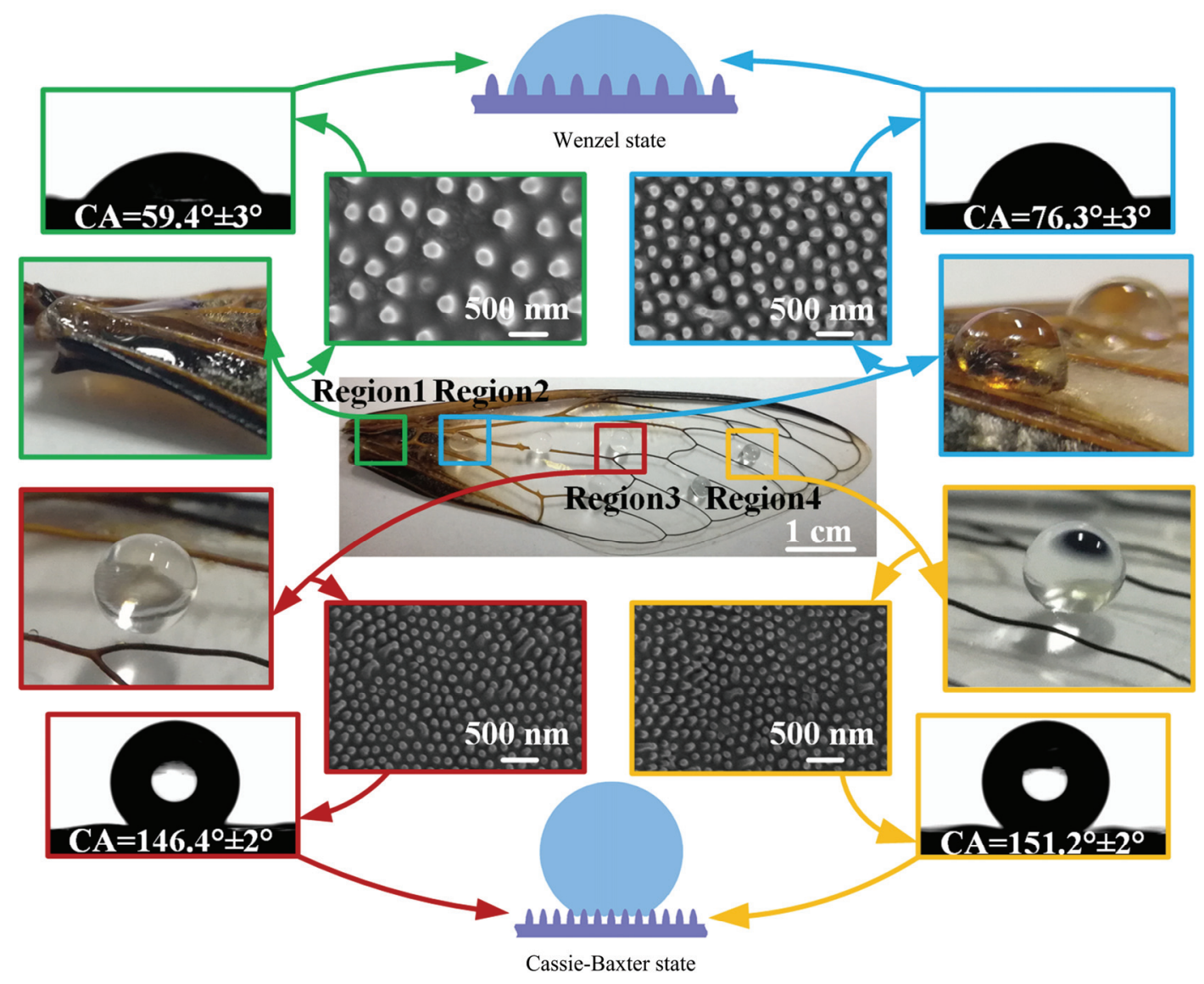

Fig. 5 SEM images for nanopillars, wetting states for $8 \mu \mathrm{L}-10 \mu \mathrm{L}$ water droplets, and contact angles for $4 \mu \mathrm{L}$ droplet at four regions along wing vein, and schematics for the Wenzel and Cassie-Baxter wetting states.

regions $1-4$ are also displayed. The height $(H)$, radius $(R)$, and gap $(G)$ of the nanopillars are determined by using the Image $\mathbf{J}$ software, and the results are listed in Table 1. At regions 1 and 2, the interspaces between the nanopillars are larger, so the droplets may easily penetrate into their bottom, exhibiting a hydrophilic Wenzel state with low CAs (Fig. 5). High adhesion to a droplet appears in the Wenzel state ${ }^{[41]}$, so the droplets impacting at regions 1 and 2 cannot bounce off (Figs. 2a and 2b). At regions 3 and 4, the packed nanopillars with small interspaces can effectively hinder the droplets from wetting the lower parts of the inter-nanopillar areas where considerable air pockets are formed. So, the solid-liquid contact area can be reduced and the droplets exhibit a hydrophobic Cassie-Baxter state with higher CAs (Fig. 5). Low adhesion to a droplet makes the droplets impacting at regions 3 and 4 bounce off (Figs. 2c and 2d).
Table 1 Parameters of nanopillars at four regions along wing vein

\begin{tabular}{ccccc}
\hline Parameter & Region 1 & Region 2 & Region 3 & Region 4 \\
\hline$H(\mathrm{~nm})$ & $628 \pm 8$ & $212 \pm 8$ & $201 \pm 6$ & $197 \pm 6$ \\
$R(\mathrm{~nm})$ & $125.3 \pm 3$ & $62.5 \pm 3$ & $44.5 \pm 3$ & $32.4 \pm 3$ \\
$G(\mathrm{~nm})$ & $301 \pm 10$ & $210 \pm 5$ & $123 \pm 3$ & $113 \pm 5$ \\
\hline
\end{tabular}

Two wetting models are established to predict the apparent CAs $\left(\theta^{*}\right)$ of water droplets at the hydrophilic and hydrophobic regions on the cicada wing. The droplets at regions 1 and 2 are in the Wenzel state, the relationship between the $\theta^{*}$ and intrinsic $\mathrm{CA}(\theta)$ for the cicada wing can be described by the following Wenzel equation $^{[42]}$ :

$$
\cos \theta^{*}=r_{\mathrm{a}} \cos \theta,
$$

where $r_{\mathrm{a}}$ is the ratio of the real contact area $\left(S_{\mathrm{a}}\right)$ of solid-liquid interface to its projected fraction $\left(S_{\mathrm{p}}\right)$ :

$$
r_{\mathrm{a}}=\frac{S_{\mathrm{a}}}{S_{\mathrm{p}}} .
$$


According to the SEM images of the nanopillars and their geometric parameters as well as the schematics for the Wenzel wetting state of the droplet (Fig. 5 and Table 1), an idealized geometric model (as shown in Fig. 6a) is developed for the nanopillars at regions 1 and 2, which is divided into many pieces of elemental areas. Figs. $6 \mathrm{~b}$ and $6 \mathrm{c}$ show the individual elemental area contacting with a water droplet. Then, the $S_{\mathrm{a}}$ and $S_{\mathrm{p}}$ can be calculated as follows:

$$
\begin{gathered}
S_{\mathrm{a}}=(G+2 R)^{2}+2 \pi R H, \\
S_{\mathrm{P}}=(G+2 R)^{2} .
\end{gathered}
$$

Thus, Eq. (4) can be rewritten as:

$$
\cos \theta^{*}=\left[1+\frac{2 \pi R H}{(G+2 R)^{2}}\right] \cos \theta .
$$

Substituting the geometric parameters of the nanopillars (Table 1) and the $\theta^{*}$ of the $\operatorname{droplet}\left(76.3^{\circ}\right.$, Fig. 5) at region 2 into Eq. (8), the $\theta$ for the cicada wing is calculated to be $81.4^{\circ}$. Then, substituting the geometric parameters of the nanopillars (Table 1) at region 1 and the calculated $\theta$ value into Eq. (8) to predict the $\theta^{*}$ of the droplet at region 1 . The predicted $\theta^{*}$ value is about $66.9^{\circ}$, which is close to the tested one $\left(59.4 \pm 3^{\circ}\right.$, Fig. 5). Thus, the proposed model shown in Fig. 6 is valid for the nanopillars at the hydrophilic region (regions 1 and 2) on the cicada wing surface.

The droplets at regions 3 and 4 are in the Cassie-Baxter state, the relationship between the $\theta^{*}$ and $\theta$ can be described by the following equation proposed by Cassie and Baxter ${ }^{[43]}$.

$$
\cos \theta^{*}=f_{\mathrm{SL}} \cos \theta-f_{\mathrm{LV}},
$$

where $f_{\mathrm{SL}}$ and $f_{\mathrm{LV}}$ are the projected fraction of solid-liquid and liquid-vapour interfaces, respectively. According to the SEM images of the nanopillars and their geometric parameters as well as the schematics for the Cassie-Baxter wetting state of the droplet (Fig. 5 and Table 1), an idealized geometric model (as shown in Fig. 7a) is developed for the nanopillars at regions 3 and 4 , and the individual elemental area contacting with a water droplet is shown in Figs. 7b and 7c. Then, for the individual elemental area, $f_{\mathrm{SL}}$ and $f_{\mathrm{LV}}$ can be expressed as:

$$
\begin{gathered}
f_{\mathrm{SL}}=\frac{2 \pi R^{2}(1+\sin \alpha)}{(G+2 R)^{2}}, \\
f_{\mathrm{LV}}=\frac{(G+2 R)^{2}-\pi(R \cos \alpha)^{2}}{(G+2 R)^{2}},
\end{gathered}
$$

where $\alpha$ is wetting angle. Thus, Eq. (9) can be rewritten as:

$$
\cos \theta^{*}=-1+\frac{2 \pi R^{2}(1+\sin \alpha) \cos \theta+\pi(R \cos \alpha)^{2}}{(G+2 R)^{2}} .
$$

Substituting the geometric parameters of the nanopillars (Table 1) and $\theta^{*}=151.2^{\circ}$ (Fig. 5) at region 4 and the aforementioned $\theta$ value for the cicada wing into Eq. (12), $\alpha$ is calculated to be $29.4^{\circ}$. Then, substituting the geometric parameters of the nanopillars at region 3 (Table 1) and the calculated $\alpha$ value into Eq. (12) to predict the $\theta^{*}$ of the droplet at region 3 . The predicted $\theta^{*}$ value is about $147.1^{\circ}$, which is close to the tested one

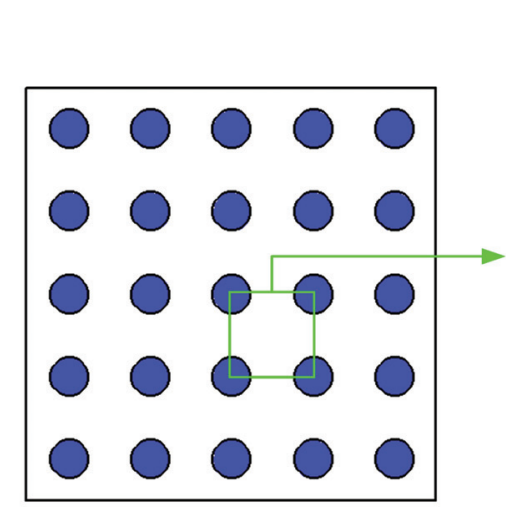

(a)

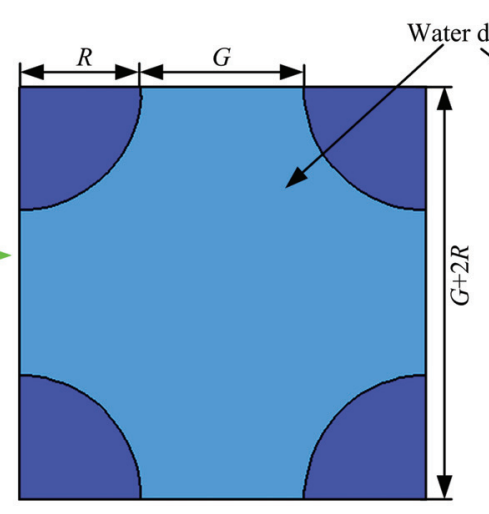

(b)

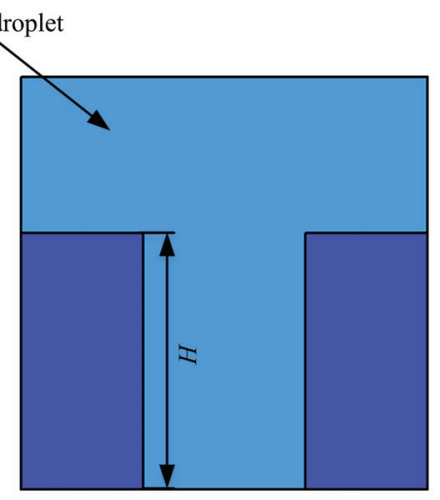

(c)

Fig. 6 Schematics of (a) top-view of idealized geometric model for nanopillars at hydrophilic region (regions 1 and 2) on cicada wing and (b) top-view and (c) side-view of individual elemental area contacting with water droplet. 


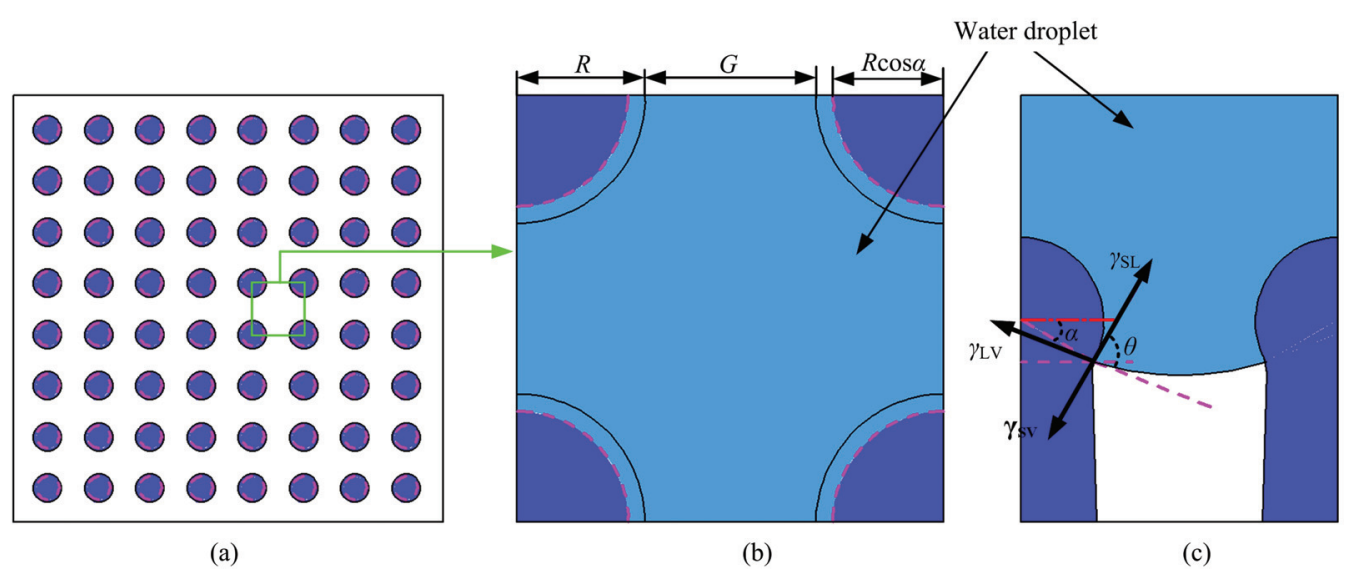

Fig. 7 Schematics of (a) top-view of idealized geometric model for nanopillars at hydrophobic region (regions 3 and 4) on cicada wing and (b) top-view and (c) side-view of individual elemental area contacting with water droplet. $\gamma_{\mathrm{SL}}, \gamma_{\mathrm{LV}}$, and $\gamma_{\mathrm{SV}}$ are solid-liquid, liquid-vapour, and solid-vapour surface tensions, respectively.

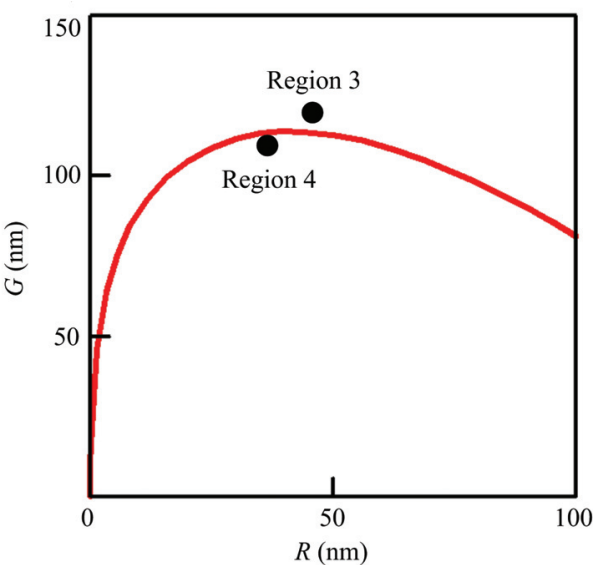

Fig. 8 Gap $(G)$-radius $(R)$ relationship curve on nanopillared surface for keeping it superhydrophobic $\left(\theta^{*}=150^{\circ}\right)$.

$\left(146.4^{\circ} \pm 2^{\circ}\right.$, Fig. 5). Thus, the proposed model shown in Fig. 7 is valid for the nanopillars at the hydrophobic region (regions 3 and 4 ) on the cicada wing surface.

A relationship between the $G$ and $R$ should be established to maintain the hydrophobic/superhydrophobic state on the nanopillared surface and so to make Eq. (12) valid. Herein, the Mahadevan-Pomeau model is used to describe the wetting behavior of a static water droplet on a non-wetting surface ${ }^{[44]}$. Based on the model shown in Fig. 7 and the geometric parameters of the nanopillars at regions 3 and 4 (Table 1), the following relationship between the $R$ and $G$ can be derivated:

$$
\frac{R}{(G+2 R)^{2}}=\frac{293436.3 \rho g r}{\pi \gamma \sin \theta^{*}\left(1+\cos \theta^{*}\right)}
$$

where $r$ is the radius of the water droplet. According to Eq. (13), the $G$ is plotted against the $R$ for keeping the nanopillared surface superhydrophobic $\left(\theta^{*}=150^{\circ}\right)$, and the result is shown in Fig. 8. As can be seen in Fig. 8, with the decrease of the $R$, the $G$ should be increased appropriately when $R \geq \sim 42 \mathrm{~nm}$, whereas the $G$ should be reduced to prevent the transition from the Cassie-Baxter wetting state to the Wenzel state when $R \leq$ $\sim 42 \mathrm{~nm}$. Moreover, the $R$ and $G$ values of the nanopillars at regions 3 and 4 (Table 1) are close to the $G-R$ relationship curve.

\section{Conclusion}

Dynamic impact tests are performed to investigate the wetting behavior of the cicada wing surface. It is found that the water droplets impacting on the surfaces of the four regions along the wing vein of cicada exhibit different dynamic responses. The gradient wetting state (from hydrophilicity to hydrophobicity and further to superhydrophobicity) exists from the foot to apex of the wing vein, which is attributed to different radius, height, and gap of the nanopillars. The hydrophobic region on the wing surface can stabilize the Cassie-Baxter state under a $288 \mathrm{~Pa}$ external pressure. According to the geometric parameters of the nanopillars at the hydrophilic and hydrophobic regions on the cicada wing, the Wenzel and Cassie-Baxter models are established to predict the apparent contact angles on the wing surface. By understanding the role of the nanopillars on the wing surface, biomimetic nanostructured surfaces with periodic or gradient wetting states can be designed and efficiently fabricated, which may be developed into multifunctional surfaces with water harvesting, water droplet transpor- 
tation, antifogging, controlled liquid movement, and self-cleaning related to the natural properties of cicada wings.

\section{Acknowledgment}

Financial support provided by the National Natural Science Foundation of China (Grant No. 51533003), the Natural Science Foundation of Guangdong Province (Grant No. 2016A030308018), the Guangzhou Municipal Science and Technology Project (Grant No. 201807010088), and the Opening Project (KFKT1805) of Key Laboratory of Polymer Processing Engineering (Ministry of Education) are gratefully acknowledged.

* All supplementary materials are available at https://doi.org/10.1007/s42235-020-0080-x.

Open Access This article is licensed under a Creative Commons Attribution 4.0 International License, which permits use, sharing, adaptation, distribution and reproduction in any medium or format, as long as you give appropriate credit to the original author(s) and the source, provide a link to the Creative Commons licence, and indicate if changes were made.

The images or other third party material in this article are included in the article's Creative Commons licence, unless indicated otherwise in a credit line to the material. If material is not included in the article's Creative Commons licence and your intended use is not permitted by statutory regulation or exceeds the permitted use, you will need to obtain permission directly from the copyright holder.

To view a copy of this licence, visit http://creativecommons.org/licenses/by/4.0/.

\section{References}

[1] Sun J Y, Bhushan B. Structure and mechanical properties of beetle wings: A review. RSC Advances, 2012, 2, 12606-12623.

[2] Zan G T, Wu Q S. Biomimetic and bioinspired synthesis of nanomaterials/nanostructures. Advanced Materials, 2016, 28, 2099-2147.

[3] Barthlott W, Mail M, Bhushan B, Koch K. Plant surfaces: Structures and functions for biomimetic innovations. Nano-Micro Letters, 2017, 9, 1265-1305.

[4] Gao X, Yan X, Yao X, Xu L, Zhang K, Zhang J, Yang B, Jiang L. The dry-style antifogging properties of mosquito compound eyes and artificial analogues prepared by soft li- thography. Advanced Materials, 2007, 19, 2213-2217.

[5] Zheng Y M, Bai H, Huang Z B, Tian X L, Nie F Q, Zhao Y, Zhai J, Jiang L. Directional water collection on wetted spider silk. Nature, 2010, 463, 640-643.

[6] Comanns P, Effertz C, Hischen F, Staudt K, Bohme W, Baumgartner W. Moisture harvesting and water transport through specialized microstructures on the integument of lizards. Beilstein Journal of Nanotechnology, 2011, 2 , 204-214.

[7] Ju J, Bai H, Zheng Y M, Zhao T Y, Fang R C, Jiang L. A multi-structural and multi-functional integrated fog collection system in cactus. Nature Communications, 2012, 3, 1247.

[8] Yamagishi R, Maeda H, Kasuga T. Water wettability dependence on surface structure of a snail shell. Bioinspiration \& Biomimetics, 2020, 15, 036001.

[9] Parker A R, Lawrence C R. Water capture by a desert beetle. Nature, 2001, 414, 33-34.

[10] Malik F T, Clement R M, Gethin D T, Krawszik W, Parker A R. Nature's moisture harvesters: A comparative review. Bioinspiration \& Biomimetics, 2014, 9, 031002.

[11] Yin X Y, Wang D A, Liu Y P, Yu B, Zhou F. Controlling liquid movement on a surface with a macro-gradient structure and wetting behavior. Journal of Material Chemistry A, 2014, 2, 5620-5624.

[12] Choo S, Choi H J, Lee H. Water-collecting behavior of nanostructured surfaces with special wettability. Applied Surface Science, 2015, 324, 563-568.

[13] Regan D P, Howell C. Droplet manipulation with bioinspired liquid-infused surfaces: A review of recent progress and potential for integrated detection. Current Opinion in Colloid \& Interface Science, 2019, 39, 137-147.

[14] Brown P S, Bhushan B. Bioinspired materials for water supply and management: Water collection water purification and separation of water from oil. Philosophical Transactions of the Royal Society A, 2016, 374, 20160135.

[15] Zhang S N, Huang J Y, Chen Z, Lai Y K. Bioinspired special wettability surfaces: From fundamental research to water harvesting applications. Small, 2017, 13, 1602992.

[16] Wang Y, Ma K, Xin J H. Stimuli-responsive bioinspired materials for controllable liquid manipulation: Principles fabrication and applications. Advanced Functional Materials, 2018, 281705128.

[17] Sun M X, Liang A P, Zheng Y M, Watson G S, Watson J A. A study of the anti-reflection efficiency of natural nano-arrays of varying sizes. Bioinspiration \& Biomimetics, 2011, 6, 026003 .

[18] Wang Z, Li B, Feng X M, Jiao Z B, Zhang J Q, Niu S C, Han 
Z W, Ren L Q. Rapid fabrication of bio-inspired antireflection film replicating from cicada wings. Journal of Bionic Engineering, 2020, 17, 34-44.

[19] Sun M, Watson G S, Zheng Y J, Watson J A, Liang A. Wetting properties on nanostructured surfaces of cicada wings. Journal of Experimental Biology, 2009, 212, 3148-3155.

[20] Pogodin S, Hasan J, Baulin V A, Webb H K, Truong V K, Nguyen T H P, Boshkovikj V, Fluke C J, Watson G S, Watson J A, Crawford R J, Ivanova E P. Biophysical model of bacterial cell interactions with nanopatterned cicada wing surfaces. Biophysical Journal, 2013, 104, 835-840.

[21] Kelleher S M, Habimana O, Lawler J, O'Reilly B, Daniels S, Casey E, Cowley A. Cicada wing surface topography: An investigation into the bactericidal properties of nanostructural features. ACS Applied Materials \& Interfaces, 2016, 8, 14966-14974.

[22] Gangadoo S, Chandra S, Power A, Hellio C, Watson G S, Watson J A, Green D W, Chapman J. Biomimetics for early stage biofouling prevention: Templates from insect cuticles. Journal of Materials Chemistry B, 2017, 4, 5747-5754.

[23] Wisdom K M, Watson J A, Qu X, Liu F, Watson G S, Chen C H. Self-cleaning of superhydrophobic surfaces by self-propelled jumping condensate. PNAS, 2013, 110, 7992-7997.

[24] Oh J, Dana C E, Hong S, Roman J K, Jo K D, Hong J W, Nguyen J, Cropek D M, Alleyne M, Miljkovic N. Exploring the role of habitat on the wettability of cicada wings. ACS Applied Materials \& Interfaces, 2017, 9, 27173-27184.

[25] Huang H X, An Y. Fabrication and condensate microdrop self-propelling of biomimetic nanostructured polymer surfaces without chemical modification. ACS Applied Polymer Material, 2019, 1, 939-943.

[26] Cai J G, Qi L M. Recent advances in antireflective surfaces based on nanostructure arrays. Materials Horizons, 2015, 2 , 37-53.

[27] Xie H, Huang H X, Mi H Y. Gradient wetting state for droplet transportation and efficient fog harvest on nanopillared cicada wing surface. Materials Letters, 2018, 221, 123-127.

[28] Zhang G M, Zhang J, Xie G Y, Liu Z F, Shao H B. Cicada wings: A stamp from nature for nanoimprint lithography. Small, 2006 2, 1440-1443.

[29] Xie G Y, Zhang G M, Lin F, Zhang J, Liu Z F, Mu S C. The fabrication of subwavelength anti-reflective nanostructures using a bio-template. Nanotechnology, 2008, 19, 095605.

[30] Xie H, Huang H X, Peng Y J. Rapid fabrication of bio-inspired nanostructure with hydrophobicity and antireflectivity on polystyrene surface replicating from cicada wings. Nanoscale, 2017, 9, 11951-11958.

[31] Kostovski G, White D J, Mitchell A, Austin M W, Stoddart P R. Nanoimprinted optical fibres: Biotemplated nanostructures for SERS sensing. Biosensors and Bioelectronics, 2009, 24, 1531-1535.

[32] Tanahashi I, Harada Y. Naturally inspired SERS substrates fabricated by photocatalytically depositing silver nanoparticles on cicada wings. Nanoscale Research Letters, 2014, 9, $1-5$.

[33] Zhang D, Kostovski G, Karnutsch C, Mitchell A. Random lasing from dye doped polymer within biological source scatters: The pomponia imperatorial cicada wing random nanostructures. Organic Electronics, 2012, 13, 2342-2345.

[34] Huang Y C, Hammitt F, Yang W. Hydrodynamic phenomena during high-speed collision between liquid droplet and rigid plane. Journal of Fluids Engineering, 1973, 95, 276-292.

[35] Zhang B, Lei Q, Wang Z K, Zhang X R. Droplets can rebound toward both directions on textured surfaces with a wettability gradient. Langmuir, 2016, 32, 346-351.

[36] Sheng X L, Zhang J H. Superhydrophobic behaviors of polymeric surfaces with aligned nanofibers. Langmuir, 2009, 25, 6916-6922.

[37] Chen A F, Huang H X. Rapid fabrication of T-shaped micropillars on polypropylene surfaces with robust cassie-baxter state for quantitative droplet collection. Journal of Physical Chemistry C, 2016, 120, 1556-1561.

[38] Tuvshindorj U, Yildirim A, Ozturk F E, Bayindir M. Robust Cassie state of wetting in transparent superhydrophobic coatings. ACS Applied Materials \& Interfaces, 2014, 6, 9680-9688.

[39] Guan W S Huang H X, Wang B. Topographic design and application of hierarchical polymer surfaces replicated by microinjection compression molding. Journal of Micromechanics and Microengineering, 2013, 23, 105010.

[40] Lafuma, Quere D. Superhydrophobic states. Nature Materials, 2003, 2, 457-460.

[41] Chen L, Xiao Z, Chan P P C, Lee Y K, Li Z. A comparative study of droplet impact dynamics on a dual-scaled superhydrophobic surface and lotus leaf. Applied Surface Science, 2011, 257, 8857-8863.

[42] Wenzel R N. Resistance of solid surfaces to wetting by water. Industrial \& Engineering Chemistry Research, 1936, 28, 988-994.

[43] Cassie A B D, Baxter S. Wettability of porous surfaces. Transacions of Faraday Society, 1944, 40, 546-551.

[44] Mahadevan L, Pomeau Y. Rolling droplets. Physics of Fluids, 1999, 11, 2449. 